\title{
Persistent Inflammation Subverts Thrombospondin- 1-Induced Regulation of Retinal Angiogenesis and Is Driven by CCR2 Ligation
}

\author{
Mei Chen, ${ }^{*}$ David A. Copland, ${ }^{\dagger}$ Jiawu Zhao, ${ }^{*}$ \\ Jian Liu, ${ }^{\dagger}$ John V. Forrester, ${ }^{\star}$ Andrew D. Dick, ${ }^{\dagger}$ \\ and Heping $\mathrm{Xu}^{\star \ddagger}$ \\ From the Centre for Vision and Vascular Science, ${ }^{*}$ Queen's \\ University Belfast, Belfast; the School of Cellular and Molecular \\ Medicine, ${ }^{\dagger}$ University of Bristol, Bristol; and Section of \\ Immunology and Infection, ${ }^{\ddagger}$ Division of Applied Medicine, \\ Institute of Medical Sciences, University of Aberdeen, Aberdeen, \\ United Kingdom
}

Neovascular retinal disease is a leading cause of blindness orchestrated by inflammatory responses. Although noninfectious uveoretinitis is mediated by $\mathrm{CD}^{+} \mathrm{T}$ cells, in the persistent phase of disease, angiogenic responses are observed, along with degeneration of the retina. Full clinical manifestation relies on myeloid-derived cells, which are phenotypically distinct from, but potentially sharing common effector responses to age-related macular degeneration. To interrogate inflammation-mediated angiogenesis, we investigated experimental autoimmune uveoretinitis, an animal model for human uveitis. After the initial acute phase of severe inflammation, the retina sustains a persistent low-grade inflammation with tissueinfiltrating leukocytes for over 4 months. During this persistent phase, angiogenesis is observed as retinal neovascular membranes that arise from inflamed venules and postcapillary venules, increase in size as the disease progresses, and are associated with infiltrating arginase- $1^{+}$macrophages. In the absence of thrombospondin-1, retinal neovascular membranes are markedly increased and are associated with arginase- $1^{-} \mathrm{CD} 68^{+}$macrophages, whereas deletion of the chemokine receptor CCR2 resulted in reduced retinal neovascular membranes in association with a predominant neutrophil infiltrate. CCR2 is important for macrophage recruitment to the retina in experimental autoimmune uveoretinitis and promotes chronicity in the form of a persistent angiogenesis response, which in turn is regulated by constitutive expression of angiogenic inhibitors like thrombospondin-1. This model offers a new platform to dissect the molecular and cellular pathology of inflammation-induced ocular angiogenesis. (Am J Pathol 2012, 180:235-245; DOI: 10.1016/j.ajpath.2011.09.020)

Retinal and choroidal neovascularization are common sight-threatening sequelae that occur in a variety of retinal diseases including age-related macular degeneration $(A M D)^{1}$ and various forms of posterior segment intraocular inflammation (PSII). ${ }^{2}$ On the basis of the site of angiogenesis, retinal neovascular membranes (RNM) are classified into subretinal choroidal neovascular membrane (CNV), in which the new blood vessels arise from the choroid and develop most commonly under the retinal pigment epithelium, causing a hemorrhagic detachment, and intraretinal neovascular membrane, or retinal angiomatous proliferation, in which the new vessels grow de novo from the retinal vasculature. ${ }^{3}$ Although only $10 \%$ to $20 \%$ of AMD patients develop CNV (so-called neovascular $\mathrm{AMD}$ or wet $\mathrm{AMD}),{ }^{4}$ it accounts for $80 \%$ to $90 \%$ of AMD-mediated severe visual impairment. ${ }^{4-6}$ In patients with PSII, the incidence of CNV differs in different types of inflammation. In a study by Brown et al, approximately $30 \%$ of multifocal choroiditis with panuveitis and $40 \%$ of punctate inner choroidopathy developed CNV. ${ }^{7}$ In other studies, 69\% to $77 \%$ of patients with punctate inner choroidopathy were found to have CNV. ${ }^{8,9} \mathrm{CNV}$ is also a common complication of serpiginous choroiditis, ${ }^{10,11}$ posterior sympathetic ophthalmia, ${ }^{12-14}$ angioid streaks, ${ }^{15-17}$ ocular toxoplasmosis, ${ }^{18,19}$ and pathological myopia. ${ }^{16,17}$ Irrespective of spe-

Funded in part by National Eye Research Centre (UK), Underwood Trust, and the Development Trust of Queen's University Belfast.

Accepted for publication September 14, 2011.

Presented in part at the Association for Research in Vision and Ophthalmology Annual Meeting, May 2010, Fort Lauderdale, FL.

Supplemental material for this article can be found at http://ajp. amjpathol.org or at doi: 10.1016/j.ajpath.2011.09.020.

Address reprint requests to Heping Xu, M.D., Ph.D., Centre for Vision and Vascular Science, School of Medicine, Dentistry and Biomedical Sciences, Queen's University Belfast, Grosvenor Road, Belfast, BT12 6BA, Northern Ireland, UK. E-mail: heping.xu@qub.ac.uk. 
cific etiology, the common denominator in these disorders is chronic low-grade inflammation or parainflammatory dysregulation of homeostasis that generates activation of innate immunity including complement activation, microglial activation, and macrophage infiltration. ${ }^{20}$

Although the management of angiogenic diseases has undergone a revolution over the past 5 to 10 years with the use of anti-vascular endothelial growth factor (VEGF) antibody, ${ }^{21}$ RNM remains a major cause of blindness in the working population. ${ }^{2}$ Perhaps not surprisingly, because VEGF is a fundamental regulator of angiogenesis responsible for pathological blood vessel growth and increased microvascular permeability, VEGF blockade has been relatively successful in the clinics. However, neutralizing VEGF activity per se does not remove the underlying drive for angiogenesis, which includes inflammation. Chronic inflammation and angiogenesis are coupled together not only in the aforementioned retinal conditions, but also in many other disorders, including rheumatoid arthritis, psoriasis, Crohn's disease, diabetes, and cancer. In rheumatoid arthritis, new blood vessels are often formed at the early stages of inflammation, resulting in increased leukocyte ingress into the synovium, ${ }^{22}$ where leukocyte-endothelial interaction may play an important role in the initiation and progression of synovial angiogenesis, ${ }^{23}$ although during cancerogenesis, the ischemic microenvironment and tumor-associated macrophages are central for tumor angiogenesis. ${ }^{24}$ In the neuroretina, however, detailed mechanisms on how angiogenesis is formed (either from the choroid or de novo from the retina) in the presence of chronic inflammation remain poorly defined.

Posterior segment intraocular inflammation is modeled in rodents by immunizing with retinal specific antigens including $S$ antigen and interphotoreceptor retinoid binding protein (IRBP) (experimental autoimmune uveoretinitis, EAU). ${ }^{25-28}$ The model system has greatly advanced our understanding of the mechanism of disease development and has provided an excellent preclinical model for testing therapeutic agents for human PSII. ${ }^{28-31}$ In EAU, although it is evident that disease clinically remits, a low-grade inflammatory retinal infiltrate (principally mononuclear cells) persists, and there is no evidence that homeostatic reconstitution in terms of cell numbers or activation status ${ }^{32,33}$ occurs up to 60 postimmunization (p.i.) days. In the present study, we provide further evidence that, after an initial acute episode, retinal inflammation persists for over 4 months and is associated with a pathological angiogenic response, ie, RNM. RNMs during EAU are initially formed from inflamed retinal venules or postcapillary venules in the inner retina, then migrate or become relocated toward the outer layers as these cellular layers degenerate, ultimately invading the retinal pigment epithelial (RPE) layer and forming chorioretinal anastomoses with focal RPE cell detachment. Although this model system mimics the development of CNV in PSII patients, the dynamic migration of neovascular membrane from the neuroretina to the RPE layer also imitates the clinical setting of the progression of retinal angiomatous proliferation. ${ }^{3}$ We further show that the angiogenic process is more aggressive in the absence of thrombospondin-1 (TSP-1) while it is reduced when CCR2-CCL2 (chemokine C-C motif ligand 2) ligation is disrupted and macrophages are relatively absent from inflamed tissue, indicating a direct regulation of angiogenesis by TSP-1 and a partial requirement for $\mathrm{CCR} 2^{+}$myeloid cells for the angiogenic response.

\section{Materials and Methods}

\section{Animals}

Eight- to 12-week-old wild-type C57BL/6 and CCR2-deficient mice (B6.126S4-Ccr2 $2^{\text {tm1lfc }} / J$, originally purchased from Jackson Laboratory, Bar Harbor, ME) ${ }^{34}$ were bred and maintained in the medical research facility of the University of Aberdeen. Thrombospondin-1-deficient (TSP $-1^{-1-}$ ) mice (C57BL/6 background) were originally from Jack Lawler ${ }^{35}$ and were maintained in the biomedical resource facility of the University of Bristol at BC10 (back-crossed 10 generations). All animals were housed under conditions outlined in the Home Office Code of Practice and according to the Home Office Regulations for Animal Experimentation (UK) and with the Association for Research in Vision and Ophthalmology statement for the use of animals in ophthalmic and vision research.

\section{EAU Induction and Clinical Evaluation}

EAU was induced using a previously described protocol. ${ }^{34,36,37}$ Briefly, the animals were immunized with 500 $\mu \mathrm{g}$ IRBP peptide 1-2038 (GPTHLFQPSLVLDMAKVLLD; Sigma-Genosys, Cambridge, UK), emulsified 1:1 in complete Freund's adjuvant (Difco Laboratories, Detroit, MI) with the addition of $2.5 \mathrm{mg} / \mathrm{mL}$ Mycobacterium tuberculosis (H37Ra; Difco Laboratories). An additional $1 \mu \mathrm{g}$ of Bordetella pertussis toxin (Health Protection Agency, Salisbury, UK) was administered intraperitoneally at the time of IRBP immunization. At different days p.i., retinal inflammation was accessed clinically using the topical endoscopic fundus image ${ }^{33,39}$ and scored using criteria described previously. ${ }^{39}$ For fluorescein angiography, mice were injected via the tail vein with $100 \mu \mathrm{L}$ of $2 \%(\mathrm{w} / \mathrm{v})$ sodium fluorescein (Sigma-Aldrich, St. Louis, MO), and images were recorded digitally using a home-built scanning laser ophthalmoscope. $^{40}$

\section{Histopathology}

Mouse eyes were collected on days 25, 80, and 120 p.i. ( $n=6$ mice at each time point) and fixed in $2.5 \%(\mathrm{w} / \mathrm{v})$ glutaraldehyde (Fisher Chemicals, Loughborough, UK) and embedded in paraffin for standard H\&E staining. The structural and infiltrating scores of retinal inflammation were graded by two independent observers using a scoring system described previously. ${ }^{41}$

\section{Confocal Microscopy of Retinal Flat Mounts}

Eyes were collected at days 25, 60, 80, and 120 p.i. ( $n=6$ to 8 mice at each time point), then fixed in $2 \%$ paraformaldehyde (Fisher Chemicals). Flat-mount 
preparation and staining were performed as previously described. ${ }^{42,43}$ Briefly, retinas were dissected and then permeabilized in $0.3 \%$ Triton X-100 in PBS, followed by $5 \%$ bovine serum albumin blocking solution. Samples were then incubated with primary antibodies at $4^{\circ} \mathrm{C}$ overnight. After being thoroughly washed, samples were incubated with second antibodies for 3 hours at room temperature in the dark. After final-step incubation, samples were washed in PBS and mounted flat on glass slides with Vectashield mounting medium (Vector Laboratories, Burlingame, CA). Images were examined with a META LSM510 confocal microscope (Carl Zeiss Microlmaging $\mathrm{GmbH}$, Gottingen, Germany). LSM Image Browser software was used for image analysis (Carl Zeiss Microlmaging $\mathrm{GmbH}$ ).

All confocal images were analyzed by the LSM Image Browser software version 4.2 (Carl Zeiss Microlmaging $\mathrm{GmbH}$ ). The size (area) of neovascular membrane was measured in merged images from a series of Z-stack images. The numbers of neovascular membrane were counted manually in low-magnification confocal images (with $5 \times$ objective lens).

\section{Immunohistochemistry}

Mouse eyes were enucleated and embedded in optimal cutting temperature compound (Thermo Fisher, Loughborough, UK), and stored at $-80^{\circ} \mathrm{C}$ until use. Serial sections (6 $\mu \mathrm{m}$ thick) were cut with a cryostat and fixed in $2 \%$ paraformaldehyde for 10 minutes. Sections were washed and then blocked with $5 \%$ bovine serum albumin for 30 minutes. Samples were incubated with rabbit anti-mouse collagen IV (1:100, AbD Secrotec) and goat anti-CCL2 (1:100; R\&D Systems, Minneapolis, MN) or rat anti-mouse VEGF (1:100; Abcam Plc., Cambridge, UK) for 1 hour, followed by fluorescein isothiocyanate-conjugated anti-rabbit IgG and Alexa Fluor 546 conjugated donkey anti-goat IgG (Invitrogen, Paisley, UK), or phycoerythrin-conjugated anti rat IgG (1: 100; BD Biosciences, Oxford, UK) for a further hour. After final washing, sections were mounted with Vectashield Mounting Media (Vector Laboratories) and examined with the META LSM510 confocal microscope as above.

\section{Cytokine Measurement}

Supernatants of splenocytes from day 25 and day 120 p.i. EAU mice $(n=6)$ cultured with or without 25 $\mu \mathrm{g} / \mathrm{mL}$ of IRBP peptide 1-20 were analyzed for tumor necrosis factor- $\alpha$, interferon (IFN)- $\gamma$, IL-2, IL-4, IL-6, IL-10, $\mathrm{IL}-12, \mathrm{IL}-17$, and IL-21 using the fluorescence-activated cell sorting cytometric bead assay kit (BD Biosciences).

\section{RNA Isolation and Reverse Transcription and Real-Time RT-PCR}

Retinas were dissected and snap-frozen in liquid nitrogen and stored at $-80^{\circ} \mathrm{C}$ until RNA extraction. Total RNA was extracted using the RNeasy mini kit (Qiagen Ltd., West Sussex, UK) following the manufacture's instruction. The quantity and quality of the RNA were determined by
NanoDrop ND-1000 spectrophotometer (Thermo Fisher). cDNA were reverse transcribed with the same amount of total RNA using the SuperScript II Reverse Transcriptase kit and random primer (Invitrogen).

Real-time RT-PCR for Tgfb, Alk1, Alk5, and Thbs 1 was performed using the LightCycler 480 system (Roche Applied Science, Mannheim, Germany) in 384-well plates using the LightCycer 480 SYBR Green Master (Roche Diagnostics $\mathrm{GmbH}$, Mannheim, Germany). Primers were designed using the Web tool "primer 3" and synthesized by Sigma-Aldrich. Primer sequences are as fellows: Alk1: forward 5'-GAAGCAGCGGGATTTGCAC-3'; reverse 5'TACCGTCCTCTGCACCAAGAA-3'; Alk5: forward 5'-TCCCAACTACAGGACCTTTTTCA-3'; reverse: 5'-GCAGTGGTAAACCTGATCCAGA-3'; Tgfb: forward: 5'-GTGTGGAGCAACATGTGGAACT-3'; reverse: 5'-GGGCTGATCCCGTTGATTTC-3'; Thbs 1: forward: 5'-TGTGGACTTCAGCGGTACCTTCTT-3'; reverse 5'-GGACTGGGTGACTTGTTTCCACAT-3'; glyceraldehyde-3-phosphate dehydrogenase (GAPDH): forward: 5'-ACTTTGTCAAGCTCATTTCC-3'; forward: 5'-ACTTTGTCAAGCTCATTTCC-3'. The expression of Ccl2 and Vegf genes were analyzed by TaqMan gene expression assay (Vegf: Mm01281449_m1. Ccl2: Mm9999056_m1). Absolute quantification and the SD method were used for data analysis. Gene expression level was normalized to the housekeeping gene GAPDH.

\section{Data and Statistical Analyses}

Real-time RT-PCR data were analyzed by one way analysis of variance multiple comparison Dunnett's test, and cytokine production by splenocytes from different days of EAU mice were compared by Student's $t$-test. EAU clinical and histological scores were compared by the Mann-Whitney test. All data were presented as mean \pm SEM. $P<0.05$ were considered statistically significant.

\section{Results}

\section{Retinal Inflammation during Different Stages of EAU}

In C57BL/6 mice, retinal inflammation developed at 10 to 14 days p.i. and peaked at 21 to 28 days p.i. (Figure 1, B and I). As the disease progressed, the severity of retinal inflammation declined, but chronic inflammation persisted for more than 120 days (Figure 11). During the acute phase (ie, days 10 to 28 p.i.), the inflammation was characterized by retinal edema, particularly around the optic disk; engorgement and sheathing of venules; and multiple infiltrates (Figure 1B). ${ }^{39}$ In the chronic stages of EAU, the fundus appeared gray or pale in color (Figure 1, $\mathrm{C}-\mathrm{H}$ ) and often displayed both active lesions and old scars (Figure 1, E and G). Active lesions included vasculitis (Figure 1, C and D) as well as multiple infiltrates in the retina (Figure 1F) and in the inferior vitreous gel (Figure 1D). Vascular sheathing/cuffing during the chronic stages of EAU appeared as relatively clear, pale sharp edges surrounding the vessels (Figure 1, C and F), com- 

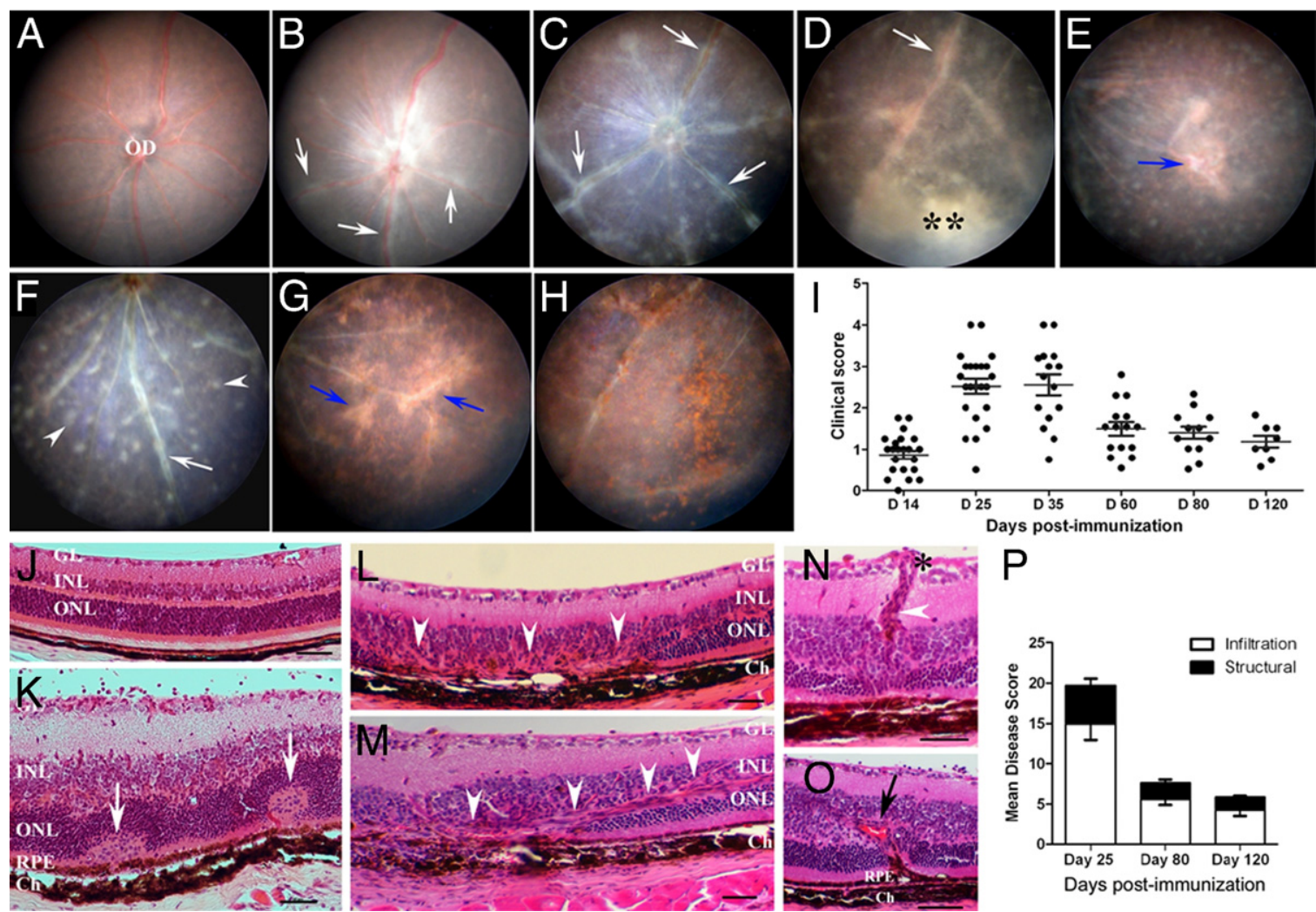

$P$
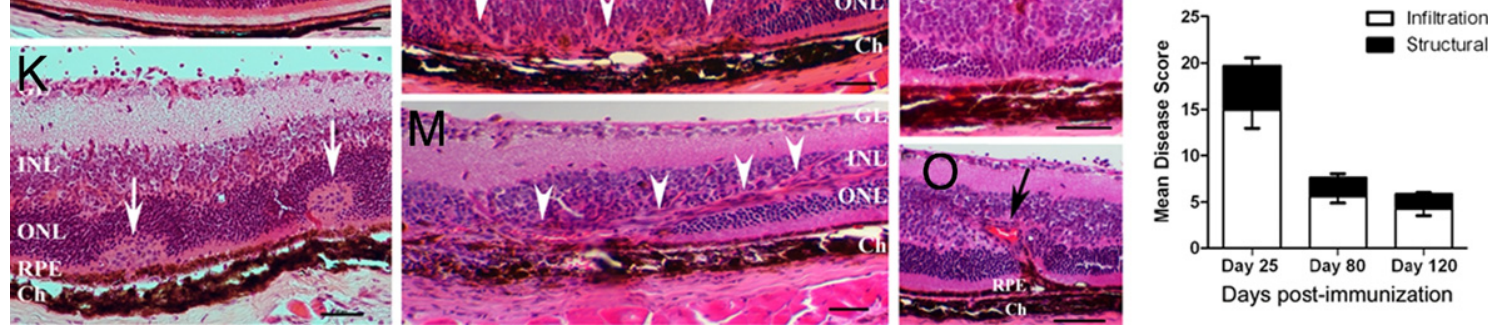

Figure 1. Clinical and histological presentations of retinal inflammation at different stages of EAU. Mice were immunized with IRBP 1-20 peptide, clinical and histological investigations (Materials and Methods) performed at different p.i. days. A: Normal nonimmunized mouse retina. OD, optic disk. B: Day 25 p.i. mouse fundus showing severe optic disk inflammation, vasculitis (white arrows). C: Day 60 p.i. mouse fundus showing retinal atrophy, small retinal infiltrates, and vascular sheathings (white arrows). D: Day 80 p.i. mouse fundus showing vascular sheathing and inferior vitreous infiltrates (asterisks). E: Day 80 p.i. mouse funds showing multiple infiltrates and an area of gliosis or scar (blue arrow). F: Day 90 p.i. mouse fundus showing multiple retinal infiltrates (arrowheads) and vascular sheathing (arrow). G: Day 120 p.i. mouse fundus showing large scars (blue arrows). H: Day 120 p.i. mouse fundus showing pigment depositions. I: Clinical score of retinal inflammation. J: H\&E staining of a retinal section from a normal mouse. K: Retinal section from a day 25 p.i. mouse showing massive inflammatory cell infiltration and retinal folds (arrows). L: Retinal section from a day 80 p.i. mouse showing structural damage and retinal scars (arrowheads). M: Retinal section from a day 120 p.i. mouse showing structural damage and a big scar extending from inner retina to outer retina (arrowheads). N: Retinal section from a day 120 p.i. mouse showing ghost vessel (asterisk) connecting with a scar (arrowhead). O: Section from a day 120 p.i. mouse showing a neovascular complex (black arrow) connecting with RPE cells. P: Histological scores (cell infiltration and structure damage) of retinal inflammation at different p.i. days. GL, ganglion layer; INL, inner nuclear layer; ONL, outer nuclear layer; RPE, retinal pigment epithelia; Ch, choroid.

pared to the blurred margins seen during the acute phase (Figure 1B), and the affected vessels often clinically appeared underperfused and consequently of reduced caliber and whitish in color (Figure 1B with Figure $1 F)$. Similarly, retinal infiltrates in the chronic phases had more distinct borders, were atrophic in appearance, and were often associated with retinal vessels (Figure 1, D and F). Occasionally, pigment deposits on the retinal surface were observed in the late stages (80 to 120 days p.i.) (Figure $1 \mathrm{H}$ ).

Fluorescein angiography of the fundus revealed an intact blood-retinal barrier in normal nonimmunized mice (see Supplemental Figure S1A at http://ajp.amjpathol. org), whereas in day 25 p.i. mice, substantial diffuse fluorescein leakage was observed in the venules and postcapillary venules, resulting in blurring of the vessel wall margins and overall obscuration of the fundal view (see Supplemental Figure S1B at http://ajp.amjpathol. org). By day 60 p.i. onward, the view of the fundus had improved, and fluorescein leakage was restricted to discrete segments of postcapillary venules (see Supplemental Figure S1, C-E, at http://ajp.amjpathol.org). The size of the hyperfluorescent lesions appeared to increase from day 60 to 120 p.i. (see Supplemental Figure S1, C-E, at http://ajp.amjpathol.org).

Histological examination showed that in day 25 p.i. mice (Figure $1 \mathrm{~K}$ ), retinal thickness increased massively compared to nonimmunized controls (Figure 1J), and infiltrating inflammatory cells were observed throughout the layers of the posterior segment of the eye (Figure $1 \mathrm{~K}$ ). Additionally, structural changes were common, with retinal folds frequently observed (Figure 1K), as well as disruption of the retinal architecture (Figure 1, $\mathrm{J}$ and $\mathrm{K}$ ). As the disease progressed toward the chronic stages, the number of infiltrating cells declined and the thickness of the retina reduced, whereupon retinal structure was partially restored (Figure 1, L and M). However, in the areas where photoreceptors were destroyed, fibrotic changes (Figure 1, L and $M$ ) were observed and retinal structure remained disordered. In addition, extensive perivascular leukocyte accumulation was observed during chronic EAU (Figure 1N). Occasionally, a ghost vessel was observed connected to a patch of gliosis, which extended from the ganglion cell layer to the outer retinal layers (Figure 1N). Interestingly, blood vessels were frequently detected at the outer layer of the retina (Figure 
A

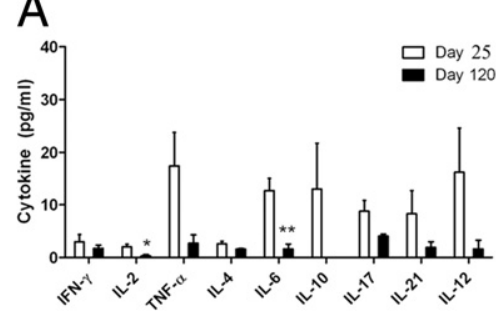

B

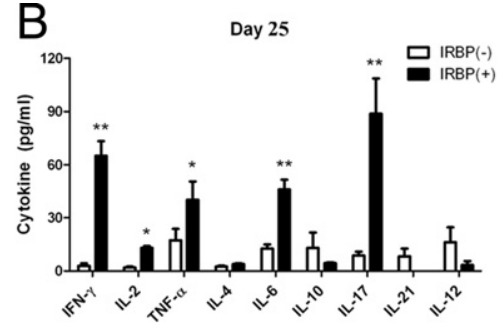

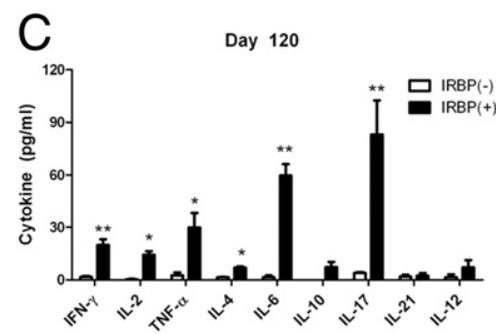

Figure 2. Cytokine production by spleen cells of day 25 and 120 p.i. mice. Splenocytes were isolated from day 25 and day 120 p.i. mice and cultured in the absence or presence of $25 \mu \mathrm{g} / \mathrm{mL}$ IRBP peptide 1-20 for 72 hours. Cytokine production in the supernatants was measurement with the cytometric bead assay kit. A: Cytokine production by cells from day 25 , and day 120 p.i. mice in the absence of IRBP stimulation. B: Cytokine production by cells from day 25 p.i. mice. C: Cytokine production by cells from day 120 p.i. mice. ${ }^{*} P<0.05$, ${ }^{* * *} P<0.01$ compared to IRBP ( - ) group. Student's $t$-test, $n=6$.

10), and they were often connected to RPE cells (Figure 10). The structural and infiltration score of EAU at day 80 and 120 p.i. mice was significantly lower than that at day 25 p.i. mice (Figure 1P), but importantly emphasized the persistence of an inflammatory response.

\section{Cytokine Production at Different Stages of EAU}

In the absence of recall responses to IRBP peptide, spleen cells from day 25 p.i. EAU mice produced small amounts of inflammatory cytokines including tumor necrosis factor- $\alpha$, IL-6, IL-17, IL-21, IL-12, and anti-inflammatory cytokine IL-10 (Figure 2A), whereas the production of these cytokines was negligible by cells from day 120 p.i. EAU mice (Figure 2A). When the cells were reactivated with $\mathrm{IRBP}$ peptide in vitro, the production of IFN- $\gamma$, IL-2, tumor necrosis factor- $\alpha, \mathrm{IL}-6$, and IL-17 was significantly increased by cells from day 25 p.i. EAU mice (Figure 2B). Cells from day 120 p.i. EAU mice also produced markedly elevated inflammatory cytokine levels after IRBP peptide stimulation in vitro (Figure 2C). However, the level of IFN- $\gamma$ production was significantly less than that of day 25 p.i. mice $(P<$ 0.05; Figure 2, B and C).

\section{Retinal Angiogenesis at Late Stages of EAU}

Confocal microscopy of retinal flat mounts using antibody to type IV collagen staining to identify vascular elements revealed no abnormal new blood vessels in day 25 p.i. EAU retina (Figure $3 \mathrm{~A}$ ). In contrast, retinas from day 60 p.i. mice showed small patches of new blood vessels (Figure 3B), and by day 80 p.i., these new vessels had formed membranous sheets (Figure 3C). New blood vessels arose directly from retinal venules (Figure 3D), and postcapillary venules formed a membrane (RNM) (Figure 3E), which was morphologically different from the surrounding normal retinal vasculature.

Z-stack scanning confocal imaging of retinal flat mounts showed that the RNM extended from the inner retina to the outer retina, where they fused with RPE cells (Figure 3F). Interestingly, patches of RPE cells were consistently associated with the RNM (Figure 3, F, G, I-K), which allowed their ready identification. The number of RNM peaked at approximately day 80 p.i. (Figure $3 \mathrm{H}$ ); however, the size of neovascular membranes continued to increase from day 80 to day 120 p.i. (Figure 3, I-L).
Because macrophages play a key role in angiogenesis, we examined the RNM complexes for macrophage infiltration. In the inflamed retinas, $\mathrm{F} 4 / 80^{+}$macrophages and $\mathrm{CD}^{+}{ }^{+} \mathrm{T}$ cells were detected in large numbers in day 25 p.i. mice (Figure 3M). However, in day 80 p.i. mice, macrophages were detected predominately surrounding and within areas of neovascular membranes (Figure $3 \mathrm{~N}$ ). Dual staining of retinal flat mounts for CD68 and arginase-1 revealed CD68 or arginase- 1 single positive cells, but no dual-positive cells (Figure 3, O-Q). From day 25 to day 120 p.i., the proportion of $\mathrm{CD}^{+} 8^{+}$cells declined, whereas the proportion of arginase- $1^{+}$cells increased (Figure 3, O-R).

\section{Growth Factors and Chemokine CCL2 Expression in the Inflamed Retina}

Preferential accumulation of inflammatory cells in the areas of neovascular membrane strongly suggests a causal role for these cells in retinal angiogenesis in this setting. To further understand the molecular mechanism, we investigated gene expression of transforming growth factor (TGF)- $\beta$, TGF- $\beta$ receptors, TSP-1, VEGF, ${ }^{44}$ and chemokine CCL2 that are likely to be produced by infiltrating macrophages and are known to be crucial in pathogenic retinal angiogenesis. ${ }^{45}$ The expression of TGF- $\beta 1$ mRNA increased over 12 -fold in the retinas from day 80 p.i. EAU mice as compared to that from nonimmunized control mice (Figure 4A). Interestingly, the expression of type 1 TGF- $\beta$ receptor ALK1 (Figure 4C), which initiates the angiogenic signaling pathway ${ }^{46}$ but not the ALK5 (Figure 4B), which initiates the antiangiogenic pathway, ${ }^{46}$ was upregulated in day 80 p.i. mice. TSP-1 is a critical enzyme for conversion of the latent form of TGF- $\beta$ into active form. The mRNA expression of Thbs 1 gene did not change in the retina of day 25 p.i. mice but significantly increased in day 80 p.i. mice (Figure 4D). There was no significant increase in expression of vegf mRNA in day 25 p.i. mouse retinas (Figure 4E); however, vegf $\mathrm{mRNA}$ expression increased 2.7 -fold in day 80 p.i. mouse retinas as compared to nonimmunized controls (Figure 4E). In addition, immunohistochemistry detected negligible levels of VEGF protein in the retina from normal nonimmunized (Figure 4F) and day 25 p.i. mice (Figure $4 G$ ), but a significant level of VEGF expression was detected in the areas of neovascular membrane in day 80 p.i. mice, particularly within the vessels (Figure $4 \mathrm{H}$ ). 

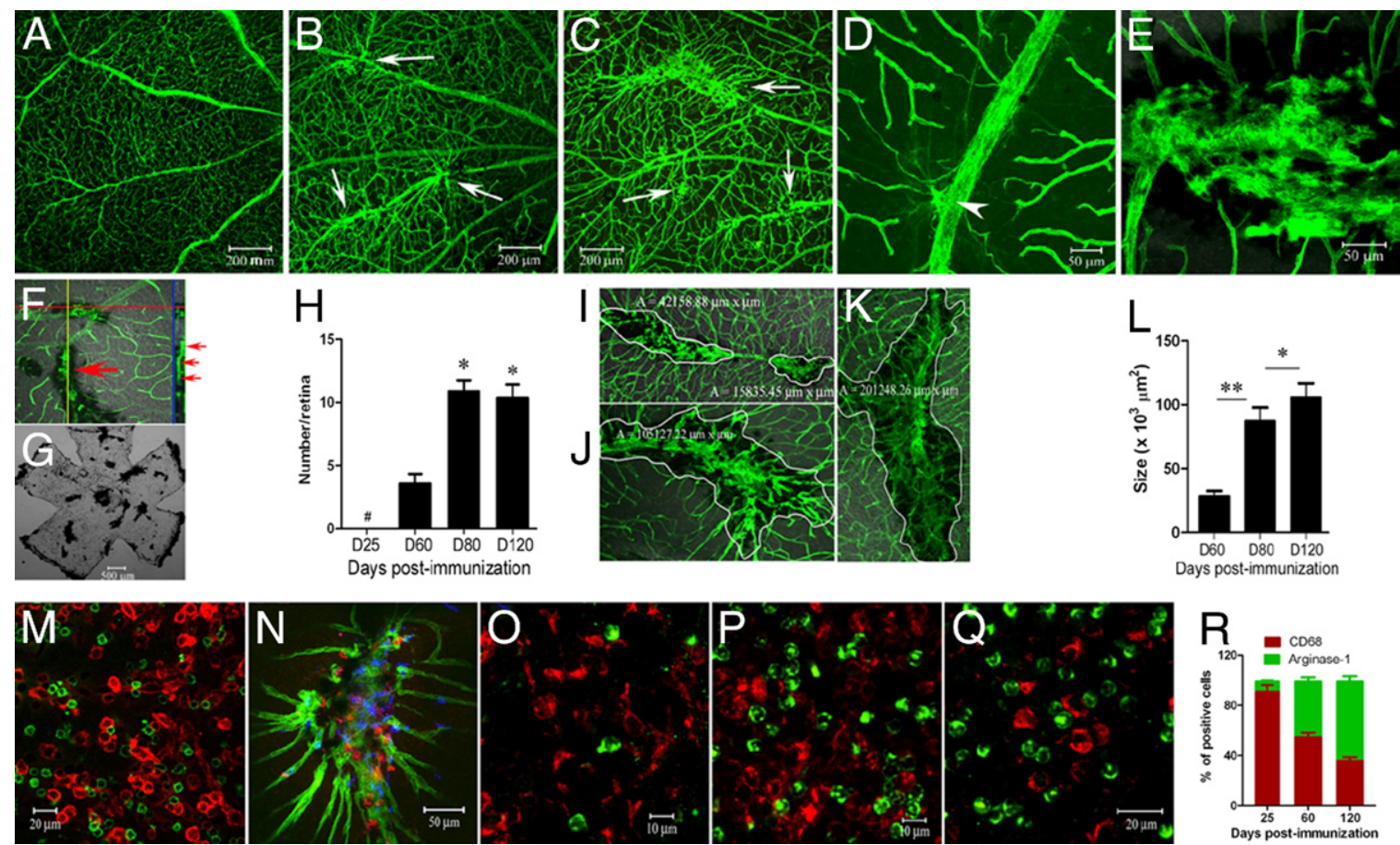

Figure 3. Confocal microscopy of retinal flat mounts at different stages of EAU. Flat-mount retinas were stained for collagen IV (A-F, I-K), or F4/80 and CD4 $(\mathbf{M}$ and $\mathbf{N})$ and collagen IV (N), or CD68 and arginase-1 (O-Q) and observed by confocal microscopy. A: Image from day 25 p.i. mouse showing normal retinal vasculature. B: Image from day 60 p.i. mouse showing new blood vessels arising from retinal venules (arrows). C: Image from day 80 p.i. mouse showing neovascular complex (arrows). D: High-magnification image from day 60 p.i. mice showing new vessels arising from retinal venules. E: Neovascular complex connecting with a postcapillary venule. F: Z-stack images from day 80 p.i. mouse showing neovascular complex (large arrow) extending from inner retina to outer retina (small arrows) causing RPE detachment (black area). G: Phase contract image of retinal flat mount from a day 80 p.i. mouse showing patches of detached RPE cells in the areas of neovascular complex. H: Number of retinal neovascular complex in mice of different days of EAU. I-K: Representative images from day $60(\mathbf{I})$, day $80(\mathbf{J})$, and day $120(\mathbf{K})$ p.i. mice showing the size of neovascular membrane. L: Average size of retinal neovascular membrane in mice of different days of EAU. ${ }^{*} P<0.05,{ }^{* *} P<0.01$ compared to day 60 p.i. mice, Dunnett's test; $n \geq 12$, no neovascular complex was detected. M: Image from day 25 p.i. mouse retina stained for F4/80 (red) and CD4 (green). N: Image from day 80 p.i. mouse retina stained for F4/80 (red), CD4 (blue), and collagen IV (green). O-Q: Images from day 25 p.i. (O), day 60 p.i. (P), and day 120 p.i. (Q) mouse retinas stained for CD68 (red) and arginase-1 (green). R: Relative proportion of CD68 ${ }^{+}$ cells and arginase- $1^{+}$cells in the retinas in different stages of EAU.

The expression of ccl2 mRNA in the retina increased 30 -fold in day 25 p.i. mice and 76 -fold in day 80 p.i. mice compared to nonimmunized control mice (Figure 4l). CCL2 protein was not detected in normal nonimmunized mouse retina (Figure $4 \mathrm{~J}$ ) but was present at significant levels in day 25 p.i. mouse retina (Figure 4K), and the expression was further increased and localized mainly around the neovascular membrane areas in day 80 p.i. mouse retinas (Figure $4 \mathrm{~L}$ ).

\section{Deletion of TSP-1 Enhances Chronic Inflammation-Mediated Retinal Angiogenesis}

Increased ALK1 but not ALK5 expression in chronic EAU suggests that TGF- $\beta$ may play a role in the angiogenic response in this setting. To further examine the role of TSP-1/TGF- $\beta 1$ in retinal angiogenesis in this model, EAU was induced in $\mathrm{TSP}_{-} 1^{-1-}$ mice. A previous study has shown that deletion of the Thbs 1 gene resulted in a more intense acute EAU. ${ }^{47}$ At day 80 p.i., wild-type (WT) (Figure $5 \mathrm{~A}$ ) and $\mathrm{TSP}^{-1-}$ (Figure $5 \mathrm{~B}$ ) mice manifested similar levels of retinal inflammation (Figure 5C). Interestingly, retinal neovascular membrane was particularly enhanced in $\mathrm{TSP}-1^{-1-}$ mice (Figure 5E) compared to WT mice (Figure 5, D and F). Unlike the WT mice, where many arginase $-1^{+}$cells were detected during late stages of EAU
(Figures 3R and $5 \mathrm{G}$ ), only few arginase $-1^{+}$cells were detected in the retinas from day 80 p.i. TSP $^{-1-}$ mice (Figure 5, $\mathrm{H}$ and I). Bone marrow-derived macrophages from $\mathrm{TSP}^{-\prime-}$ mice, however, demonstrated equal ability as WT bone marrow-derived macrophages to respond to either M1 or $\mathrm{M} 2$ conditioning, and to express nitric oxide (NO), upregulate nitric oxide synthase 2 (NOS2) or Arg1 or the Tgfb gene, and generate VEGF (see Supplemental Figure S2 at $h t t p: / /$ ajp.amjpathol.org).

\section{Deletion of CCR2 Reduces Chronic Inflammation-Mediated Retinal Angiogenesis}

The CCL2-CCR2 pathway is known to be important for macrophage and hematopoietic stem cell recruitment to sites of inflammation. ${ }^{48} \mathrm{~A}$ previous study has shown that this pathway is involved in angiogenesis, ${ }^{45}$ and currently we have enumerated high levels and localized expression of CCL2 during the chronic phase of EAU. Previous studies have shown that deletion of CCR2 does not affect the expression of EAU ${ }^{49,50}$ but may modify the nature of the inflammatory cell infiltrate (eg, neutrophils, but not macrophages, dominate the inflamed retina $\left.{ }^{49,50}\right)$. In this study, at day 80 p.i., we observed no significant difference in EAU inflammatory activity between WT and CCR2 knockout mice (Figure 6A). Histologically, fibrotic scar- 
ring was frequently observed in the retina of WT mice (Figure 6, B and C). Such changes were less frequent in CCR2 knockout mice (Figure 6, D and E). Retinal flat-mount investigation $(n=6)$ demonstrated significantly less retinal neovascular membrane in CCR2-deficient mice as compared to WT mice (Figure 6, F-H). In addition, the size of retinal neovascular membrane in CCR2 knockout mice was significantly smaller than those observed in WT mice (Figure 6, I-K). Dual staining of day 80 p.i. mouse retina for major histocompatibility complex-II and Gr-1 (granulocyte differentiation antigen-1) revealed many Gr-1 ${ }^{+}$cells in CCR2 knockout mice (Figure 6M). WT mouse retina was filled with major histocompatibility complex- $\mathrm{II}^{+} \mathrm{Gr}-1^{-}$cells (Figure 6L).

\section{Discussion}

Here, we showed that retinal inflammation from EAU in C57BL/6 does not spontaneously resolve. After the initial acute inflammation (10 to 28 days p.i.), chronic disease persists for over 4 months. Of more significance is that we show that chronic inflammation in EAU induces retinal angiogenesis. Unlike other physiological (female reproductive system) or pathological conditions (carcinogenesis, wound healing) of vessel growth, new blood vessels in chronic EAU arise from the inflamed venules and postcapillary venules in the inner retina forming vascularized membranes, which morphologically differ from normal vasculature. Once formed in the inner retina, the neovascular membranes then migrate toward the outer retina, causing RPE detachment. The model therefore mirrors inflammatory CNV observed in patients with long-standing chronic choroiditis ${ }^{2}$ and provides a unique opportunity to dissect the role of inflammation as a cause of angiogenesis and the mechanism underlying how inflammation drives - or indeed may control-pathological ocular angiogenesis.

$\mathrm{EAU}$ is a CD4 ${ }^{+}$T-cell-mediated autoimmune disease mediated by both Th1 and Th17 cells. ${ }^{51,52}$ What causes the inflammation to persist is not known. In the C57BL/6 model, inflammation is initiated by IRBP peptide 1-20specific $\mathrm{CD}^{+} \mathrm{T}$ cells and IRBP antigen in photoreceptors remains the primary target. Although many photoreceptors are damaged during the acute phase of EAU, areas where normal, healthy photoreceptors remain detectable in the chronic stages in this mouse model (Figure 1). The remaining IRBP (auto)antigen in undamaged photoreceptors is likely to be one of the continued drivers for persistence of inflammation, along with antigen and epitope spreading. Indeed, spleen antigen-specific memory $\mathrm{T}$-cell responses can be elicited late in the disease (Figure 2), although interestingly, this has shifted more toward a Th17 rather than a Th1 response.
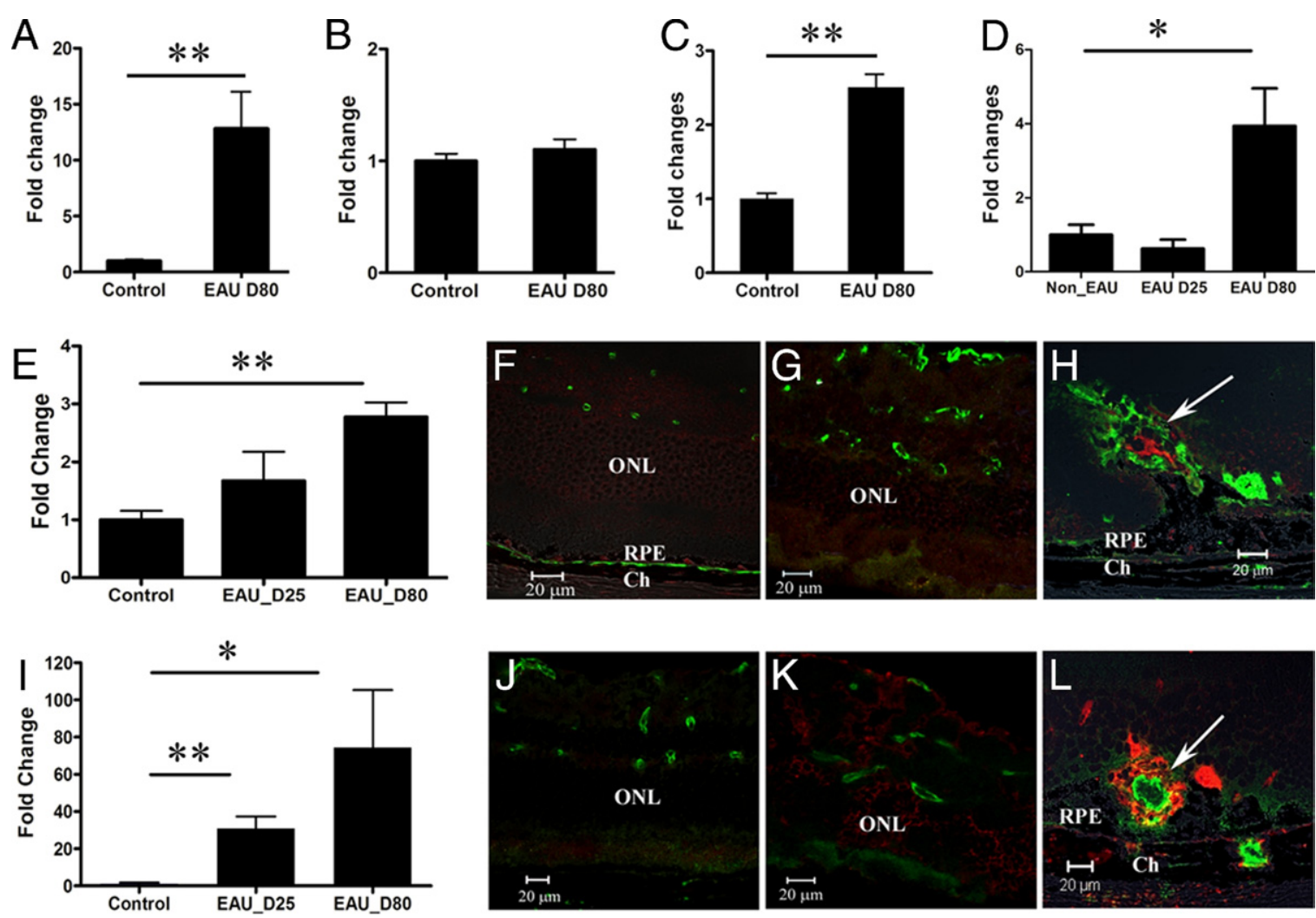

Figure 4. Expression of growth factors in mouse retina during different stages of EAU. The expression levels of mRNA in mouse retinas were determined by real-time RT-PCR, and the protein levels were examined by confocal microscopy. A: TGF- $\beta 1$ mRNA expression. B: ALK5 mRNA expression. C: ALK1 mRNA expression. D: TSP mRNA expression. E: VEGF mRNA expression. VEGF (red) and collagen IV (green) expression in the retina of normal (F), day 25 p.i. (G), and day 80 p.i. (H) mouse. I: CCL2 mRNA expression. CCL2 protein (red) and collagen IV (green) expression in normal (J), day 25 p.i. (K), and day 80 p.i. (L) mouse. ONL, outer nuclear layer; RPE, retinal pigment epithelia; Ch, choroid. Arrows, retinal neovascular complex. ${ }^{*} P<0.05$, ${ }^{* *} P<0.01$, Student's $t$-test $(\mathbf{A}-\mathbf{D})$ and Dunnett's multiple comparison test (E and $\mathbf{I}) ; n=6$ to 8 . 

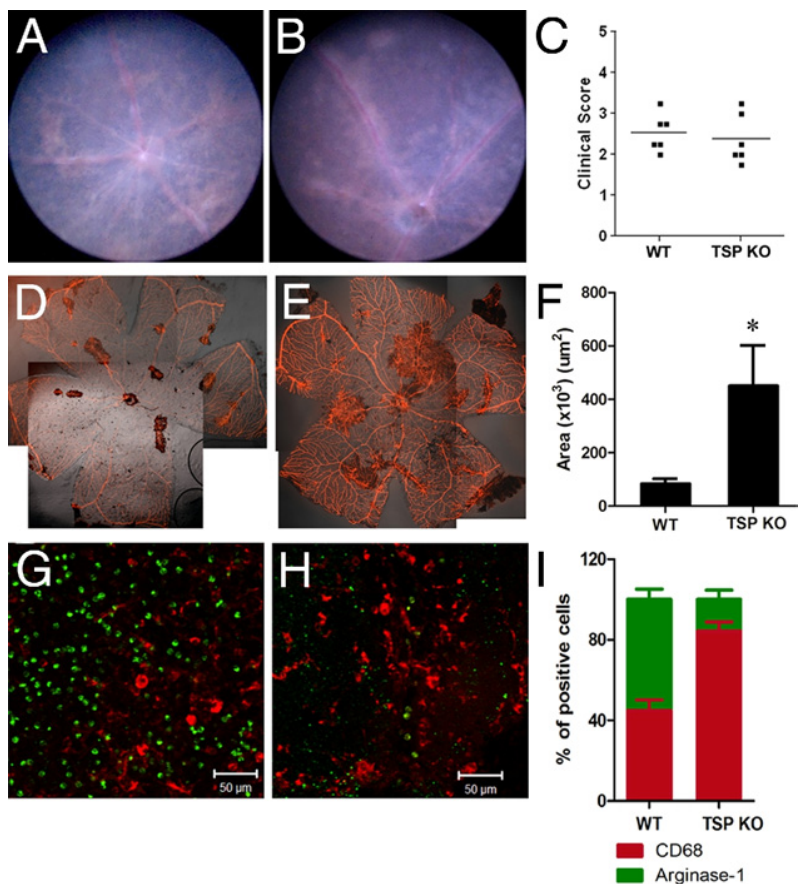

Figure 5. Retinal neovascular membrane in $\mathrm{TSP}^{-/-}$mice. EAU was induced in WT and $\mathrm{TSP}^{-1-}$ mice. Clinical inflammation was examined by topical endoscopic fundus imaging. At day 80 p.i., mice were sacrificed and retinal flat mounts were stained for collagen IV and observed by confocal microscopy. Retinal inflammation in a day 80 p.i. WT $(\mathbf{A})$ and a $\mathrm{TSP}^{-/-}$mouse (B). C: Clinical score of retinal inflammation at day 80 p.i. Confocal image of retinal flat mount from a day 80 p.i. WT (D) and $\mathrm{TSP}^{-1-}$ (E) mouse. F: Histogram showing the size of retinal neovascular membrane in WT and $\mathrm{TSP}^{-1-}$ mice. ${ }^{*} P<0.05$, unpaired Student's $t$-test, $n=6$. Confocal images of retinal flat mounts showing $\mathrm{CD}^{+} 8^{+}$(red) and arginase- $1^{+}$(green) cells in a day 80 p.i. WT (G) and a day 80 p.i. TSP- $1^{-/-}(\mathbf{H})$ mouse. I: Histogram graph showing the relative proportion of $\mathrm{CD}_{6} 8^{+}$and arginase $-1^{+}$cells in day 80 p.i. WT and TSP $-1^{-/-}$mice $(n=4)$.

The macrophage infiltration associated with the late angiogenic response also shows interesting phenotypic change. In the acute stage of the disease (day 25 p.i.), infiltrating macrophages were $\mathrm{CD}^{+}{ }^{+}$arginase-1- ${ }^{-}$, whereas in the later stages, there was a substantial in- crease in $\mathrm{Arg}-1^{+}$macrophages. In other studies, it has been shown that extent of autoimmune disease is therefore limited in later stages by the generation of regulatory networks, including regulatory $\mathrm{T}$ cells and myeloid-derived suppressor cells. ${ }^{32}$ In the current model of EAU, regulatory mechanisms appear only to have partial effect because although the acute phase subsides, the disease persists as a chronic inflammation, is associated with a predominance of Th17 $\mathrm{T}$ cells, and presents with an arginase $-1^{+}$macrophage-infiltrated foci of neovascularization and fibrosis in the form of neovascular membranes. Persistence of Th17 cells over Th1 cells in chronic inflammatory conditions has been observed in a number of settings, including tuberculosis, ${ }^{53}$ arthritis ${ }^{54,55}$ and colitis. ${ }^{56}$ Indeed, IL-17 is linked to inhibition of apoptosis which would promote resolution, and so this cytokine helps maintain inflammation. ${ }^{54,55}$

Although EAU in the late stages persists as a chronic disease, the fibrovascular "healing" response in the retina may represent an attempt at regulation of inflammation and retinal damage. Photoreceptors, blood vessels, and other retinal cells/molecules are damaged either directly by antigen-specific $\mathrm{T}$ cells or indirectly by inflammatory mediators during the acute phase of EAU. The increase in highly phagocytic arginase- $1^{+}$ macrophages (putative alternatively activated M2 macrophages ${ }^{57}$ ) is consistent with a shift in outcome from tissue damage to tissue repair and removal of damaged or dead cells/molecules during the wound healing process. ${ }^{58}$ Arginase- 1 is involved in proline and polyamine biosynthesis; the former promote extracellular matrix membrane construction, whereas the latter are involved in cell proliferation. ${ }^{59}$ In addition, retinal tissues from day 80 p.i. EAU mice also expressed high levels of TGF- $\beta 1$ and VEGF (Figure 4), further corroborating a drive toward healing rather than destructive inflammation.

Specific molecular mechanisms almost certainly direct retinal inflammation in late-stage EAU toward a
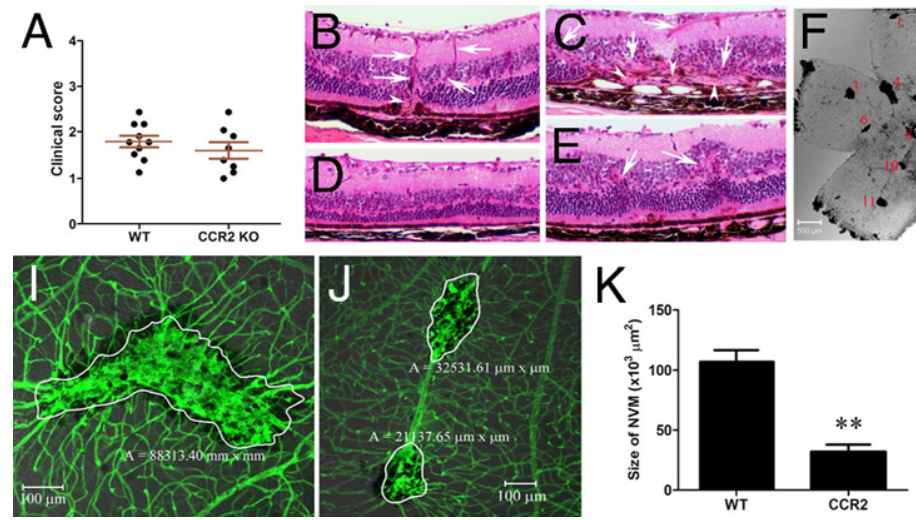

Figure 6. Retinal neovascular membrane in CCR2-deficient mice. EAU was induced in WT and CCR2 knockout mice. At day 80 p.i., mice were sacrificed and retinal flat mounts were prepared for confocal microscopy. A: Clinical score at day 80 p.i. B and C: Histology of EAU retina from day 80 p.i. WT mice. D and E: Histology of EAU retina from day 80 p.i. CCR $2^{-/-}$mice. Phase contrast images of retinal flat mounts from a WT mouse (F) and a CCR2 knockout mouse (G). Numbered pigmented areas represent retinal neovascular membrane with RPE detachment. H: Average number of retinal neovascular membrane in WT and CCR2 knockout mice at day 80 p.i. ${ }^{* *} P<0.01$, unpaired Student's $t$-test; $n=12$ eyes. Representative confocal images showing the size of neovascular membrane in WT (I) and CCR2 knockout ( $\mathbf{J})$ mice. K: Average size of neovascular membrane in WT and CCR2 knockout mice. ${ }^{* * *} P<0.01$, unpaired Student's $t$-test, $n=12$ eyes. Confocal images showing major histocompatibility complex-II ${ }^{+}$(green) and $G r-1^{+}$(red) cells in day 80 p.i. WT (L) and CCR2 knockout (M) mice. 
wound healing response. Angiogenesis is an intrinsic component of the wound healing process, and macrophages are central to this process. Here we have shown that the late-stage EAU retinal angiogenic response and the macrophage infiltration are significantly reduced in the absence of CCR2-CCL2 ligation. CCR2 is expressed on inflammatory macrophages and is a critical receptor involved in recruitment of macrophages to sites of inflammation, ${ }^{60,61}$ including the retina in EAU. ${ }^{37}$ Clearly, CCR2 is involved in the full expression of late-stage EAU retinal angiogenesis. Macrophage-induced angiogenesis has the potential to be fine-tuned. While "inflammatory" macrophages (M1) are robust producers of inflammatory cytokines involved in tissue damage, including VEGF, and so have angiogenic potential, ${ }^{62,63}$ "regulatory" macrophages (M2) have significantly less proinflammatory effect while still having the capacity to produce VEGF. The balance between M1 and M2 macrophages is thus shifted in late EAU toward, but not solely, angiogenesis. In CCR2deficient mice, where macrophage numbers are sparse and the infiltrate is predominantly neutrophilic (Figure 6), the angiogenic response is concomitantly reduced.

Healing responses, however beneficial they are to the survival of the host, may be damaging to delicate ocular tissues. Scar tissue in particular is a major threat to ocular function. ${ }^{64}$ The eye, and especially the retina, secretes many immunomodulatory molecules, which contribute to immunological privilege ${ }^{65}$ and include growth factors such as TGF- $\beta^{66,67}$ and TSP-1. TSP- 1 has been shown to be important in regulating the severity of the acute response in $E A U^{47}$ and is produced constitutively by retinal cells such as RPE, presumably to mediate this regulatory role. We show here that TSP-1 is also important in regulating the retinal angiogenic response because in its absence, persistent inflammation mediated retinal angiogenesis is markedly increased (Figure 5). It would thus appear that whereas inflammatory and, later, proangiogenic macrophages are necessary for the full expression of EAU and in particular for its progression to chronicity, TSP-1, in concert with many other local regulatory factors, attempts to exert control over the damaging effects of inflammation and modulate its outcome. In the absence of TSP-1, although macrophages are able to respond to M1 (lipopolysaccharide and IFN- $\gamma$ ) and M2 (IL-4, IL-13, and prostaglandin E2) conditioning and differentiate into NOS2, or arginase-1-expressing cells in vitro (see Supplemental Figure S2 at http://ajp.amjpathol.org), in the inflamed retina of late-stage EAU, macrophage infiltrates have remained inflammatory phenotype $\left(\mathrm{CD} 8^{+}\right.$arginase- $1^{-} \mathrm{M} 1$ type macrophage) and are angiogenic. ${ }^{62,63}$ The overall outcome, therefore, is increased angiogenesis as a result of the lack of constitutive retinal TSP-1.

In summary, we have described here a new model of chronic inflammation in the retina in the C57BL6 mouse. The model is characterized by persistent infiltration of arginase- $1^{+}$CD68 macrophages and discrete sheet-like foci of membranous neovascular complexes. This retina-specific angiogenic response requires CCR2-CCL2 ligations to promote macro- phage recruitment to the lesions and is regulated by tissue expression of the angiogenic inhibitor TSP-1. This model closely resembles the inflammation-induced angiogenesis that occurs in human posterior segment intraocular inflammation, and investigation of its molecular mechanisms will provide insight into the pathogenesis of ocular angiogenesis generally.

\section{Acknowledgments}

We thank Elizabeth Muckersie and Gillian Smith for technical support.

\section{References}

1. Coleman HR, Chan CC, Ferris FL 3rd, Chew EY: Age-related macular degeneration. Lancet 2008, 372:1835-1845

2. Dhingra N, Kelly S, Majid MA, Bailey CB, Dick AD: Inflammatory choroidal neovascular membrane in posterior uveitis-pathogenesis and treatment. Indian J Ophthalmol 2010, 58:3-10

3. Yannuzzi LA, Negrao S, lida T, Carvalho C, Rodriguez-Coleman $H$ Slakter J, Freund KB, Sorenson J, Orlock D, Borodoker N: Retinal angiomatous proliferation in age-related macular degeneration. Retina $2001,21: 416-434$

4. Klein R, Peto T, Bird A, Vannewkirk MR: The epidemiology of age-related macular degeneration. Am J Ophthalmol 2004, 137: $486-495$

5. Augood CA, Vingerling JR, de Jong PT, Chakravarthy U, Seland J, Soubrane G, Tomazzoli L, Topouzis F, Bentham G, Rahu M, Vioque J, Young IS, Fletcher AE: Prevalence of age-related maculopathy in older Europeans: the European Eye Study (EUREYE). Arch Ophthalmol 2006, 124:529-535

6. Owen CG, Fletcher AE, Donoghue M, Rudnicka AR: How big is the burden of visual loss caused by age related macular degeneration in the United Kingdom? Br J Ophthalmol 2003, 87:312-317

7. Brown J, Jr, Folk JC, Reddy CV, Kimura AE: Visual prognosis of multifocal choroiditis, punctate inner choroidopathy, and the diffuse subretinal fibrosis syndrome. Ophthalmology 1996, 103: $1100-1105$

8. Kedhar SR, Thorne JE, Wittenberg S, Dunn JP, Jabs DA: Multifocal choroiditis with panuveitis and punctate inner choroidopathy: comparison of clinical characteristics at presentation. Retina 2007, 27:11741179

9. Thorne JE, Wittenberg S, Jabs DA, Peters GB, Reed TL, Kedhar SR, Dunn JP: Multifocal choroiditis with panuveitis incidence of ocular complications and of loss of visual acuity. Ophthalmology 2006, 113:2310-2316

10. Laatikainen L, Erkkila $\mathrm{H}$ : Subretinal and disc neovascularisation in serpiginous choroiditis. Br J Ophthalmol 1982, 66:326-331

11. Jampol LM, Orth D, Daily MJ, Rabb MF: Subretinal neovascularization with geographic (serpiginous) choroiditis. Am J Ophthalmol 1979, 88:683-689

12. Gupta V, Gupta A, Dogra MR: Posterior sympathetic ophthalmia: a single centre long-term study of 40 patients from North India. Eye (Lond) 2008, 22:1459-1464

13. Borkowski LM, Weinberg DV, Delany CM, Milsow L: Laser photocoagulation for choroidal neovascularization associated with sympathetic ophthalmia. Am J Ophthalmol 2001, 132:585-587

14. Bernasconi O, Auer C, Zografos L, Herbort CP: Indocyanine green angiographic findings in sympathetic ophthalmia. Graefes Arch Clin Exp Ophthalmol 1998, 236:635-638

15. Chang LK, Spaide RF, Brue C, Freund KB, Klancnik JM Jr, Slakter JS: Bevacizumab treatment for subfoveal choroidal neovascularization from causes other than age-related macular degeneration. Arch Ophthalmol 2008, 126:941-945

16. Miller DG, Singerman LJ: Vision loss in younger patients: a review of choroidal neovascularization. Optom Vis Sci 2006, 83:316-325

17. Spaide RF: Choroidal neovascularization in younger patients. Curr Opin Ophthalmol 1999, 10:177-181 
18. Gaynon MW, Boldrey EE, Strahlman ER, Fine SL: Retinal neovascularization and ocular toxoplasmosis. Am J Ophthalmol 1984, 98:585-589

19. Fine SL, Owens SL, Haller JA, Knox DL, Patz A: Choroidal neovascularization as a late complication of ocular toxoplasmosis. Am J Ophthalmol 1981, 91:318-322

20. $\mathrm{Xu} \mathrm{H}$, Chen M, Forrester JV: Para-inflammation in the aging retina. Prog Retin Eye Res 2009, 28:348-368

21. Ferrara N: Vascular endothelial growth factor: basic science and clinical progress. Endocr Rev 2004, 25:581-611

22. Szekanecz Z, Koch AE: Targeting angiogenesis in rheumatoid arthritis. Curr Rheumatol Rev 2008, 4:298-303

23. Szekanecz Z, Besenyei T, Paragh G, Koch AE: New insights in synovial angiogenesis. Joint Bone Spine 2010, 77:13-19

24. Dirkx AE, Oude Egbrink MG, Wagstaff J, Griffioen AW: Monocyte/ macrophage infiltration in tumors: modulators of angiogenesis. J Leukoc Biol 2006, 80:1183-1196

25. Caspi RR: Experimental autoimmune uveoretinitis_rat and mouse. Autoimmune Disease Models: A Guidebook, Cohen IR and Miller A (Eds), Academic Press 1994, pp 57-81

26. Caspi RR, Chan CC, Wiggert B, Chader GJ: The mouse as a model of experimental autoimmune uveoretinitis (EAU). Curr Eye Res 1990 9(Suppl):169-174

27. Forrester JV, Liversidge J, Dua HS, Towler H, McMenamin PG: Comparison of clinical and experimental uveitis. Curr Eye Res 1990, 9(Suppl):75-84

28. Forrester JV, Liversidge J, Dua HS, Dick A, Harper F, McMenamin PG: Experimental autoimmune uveoretinitis: a model system for immunointervention: a review. Curr Eye Res 1992, 11(Suppl): 33-40

29. Forrester JV, Xu H, Kuffova L, Dick AD, McMenamin PG: Dendritic cel physiology and function in the eye. Immunol Rev 2010, 234:282-304

30. Caspi RR: Immunogenetic aspects of clinical and experimental uveitis. Reg Immunol 1992, 4:321-330

31. Dick AD: Immune mechanisms of uveitis: insights into disease pathogenesis and treatment. Int Ophthalmol Clin 2000, 40:1-18

32. Kerr EC, Raveney BJ, Copland DA, Dick AD, Nicholson LB: Analysis of retinal cellular infiltrate in experimental autoimmune uveoretinitis reveals multiple regulatory cell populations. J Autoimmun 2008, 31:354-361

33. Copland DA, Wertheim MS, Armitage WJ, Nicholson LB, Raveney BJ, Dick AD: The clinical time-course of experimental autoimmune uveoretinitis using topical endoscopic fundal imaging with histologic and cellular infiltrate correlation. Invest Ophthalmol Vis Sci 2008, 49: 5458-5465

34. Chen M, Muckersie E, Luo C, Forrester JV, Xu H: Inhibition of the alternative pathway of complement activation reduces inflammation in experimental autoimmune uveoretinitis. Eur J Immunol 2010, 40: 2870-2881

35. Lawler J, Sunday M, Thibert V, Duquette M, George EL, Rayburn H, Hynes RO: Thrombospondin-1 is required for normal murine pulmonary homeostasis and its absence causes pneumonia. J Clin Invest 1998, 101:982-992

36. Xu H, Dawson R, Crane IJ, Liversidge J: Leukocyte diapedesis in vivo induces transient loss of tight junction protein at the blood-retina barrier. Invest Ophthalmol Vis Sci 2005, 46:2487-2494

37. Xu H, Manivannan A, Dawson R, Crane IJ, Mack M, Sharp P, Liversidge J: Differentiation to the CCR2 + inflammatory phenotype in vivo is a constitutive. Time-limited property of blood monocytes and is independent of local inflammatory mediators. J Immunol 2005, 175: 6915-6923

38. Avichezer D, Silver PB, Chan CC, Wiggert B, Caspi RR: Identification of a new epitope of human IRBP that induces autoimmune uveoretinitis in mice of the $\mathrm{H}-2 \mathrm{~b}$ haplotype. Invest Ophthalmol Vis Sci 2000 , 41:127-131

39. Xu H, Koch P, Chen M, Lau A, Reid DM, Forrester JV: A clinical grading system for retinal inflammation in the chronic model of experimental autoimmune uveoretinitis using digital fundus images. Exp Eye Res 2008, 87:319-326

40. Xu H, Manivannan A, Goatman KA, Liversidge J, Sharp PF, Forrester $\mathrm{JV}$, Crane IJ: Improved leukocyte tracking in mouse retinal and choroidal circulation. Exp Eye Res 2002, 74:403-410

41. Dick AD, Cheng YF, Liversidge J, Forrester JV: Immunomodulation of experimental autoimmune uveoretinitis: a model of tolerance induction with retinal antigens. Eye 1994, 8(Pt 1):52-59
42. Xu H, Dawson R, Forrester JV, Liversidge J: Identification of novel dendritic cell populations in normal mouse retina. Invest Ophthalmol Vis Sci 2007, 48:1701-1710

43. Xu H, Forrester JV, Liversidge J, Crane IJ: Leukocyte trafficking in experimental autoimmune uveitis: breakdown of blood-retinal barrier and upregulation of cellular adhesion molecules. Invest Ophthalmo Vis Sci 2003, 44:226-234

44. Ishida S, Usui T, Yamashiro K, Kaji Y, Amano S, Ogura Y, Hida T, Oguchi Y, Ambati J, Miller JW, Gragoudas ES, Ng YS, D’Amore PA, Shima DT, Adamis AP: VEGF164-mediated inflammation is required for pathological, but not physiological, ischemia-induced retinal neovascularization. J Exp Med 2003, 198:483-489

45. Salcedo R, Ponce ML, Young HA, Wasserman K, Ward JM, Kleinman HK, Oppenheim JJ, Murphy WJ: Human endothelial cells express CCR2 and respond to MCP-1: direct role of MCP-1 in angiogenesis and tumor progression. Blood 2000, 96:34-40

46. Byfield SD, Roberts AB: Lateral signaling enhances TGF-beta response complexity. Trends Cell Biol 2004, 14:107-111

47. Zamiri P, Masli S, Kitaichi N, Taylor AW, Streilein JW: Thrombospondin plays a vital role in the immune privilege of the eye. Invest Ophthalmol Vis Sci 2005, 46:908-919

48. Tsou CL, Haskell CA, Charo IF: Tumor necrosis factor-alpha-converting enzyme mediates the inducible cleavage of fractalkine. J Biol Chem 2001, 276:44622-44626

49. Dagkalis A, Wallace C, Xu H, Liebau S, Manivannan A, Stone MA, Mack M, Liversidge J, Crane IJ: Development of experimental autoimmune uveitis: efficient recruitment of monocytes is independent of CCR2. Invest Ophthalmol Vis Sci 2009, 50:4288-4294

50. Sonoda KH, Yoshimura T, Egashira K, Charo IF, Ishibashi T: Neutrophil-dominant experimental autoimmune uveitis in CC-chemokine receptor 2 knockout mice. Acta Ophthalmol 2011, 89:e180e188

51. Luger D, Silver PB, Tang J, Cua D, Chen Z, Iwakura Y, Bowman EP Sgambellone NM, Chan CC, Caspi RR: Either a Th17 or a Th1 effector response can drive autoimmunity: conditions of disease induction affect dominant effector category. J Exp Med 2008, 205:799-810

52. Amadi-Obi A, Yu CR, Liu X, Mahdi RM, Clarke GL, Nussenblatt RB, Gery I, Lee YS, Egwuagu CE: TH17 cells contribute to uveitis and scleritis and are expanded by IL-2 and inhibited by IL-27/STAT1. Nat Med 2007, 13:711-718

53. Torrado E, Cooper AM: IL-17 and Th17 cells in tuberculosis. Cytokine Growth Factor Rev 2010, 21:455-462

54. Toh ML, Kawashima M, Hot A, Miossec P, Miossec P: Role of IL-17 in the Th1 systemic defects in rheumatoid arthritis through selective IL-12Rbeta2 inhibition. Ann Rheum Dis 2010, 69:1562-1567

55. Toh ML, Gonzales G, Koenders MI, Tournadre A, Boyle D, Lubberts E, Zhou Y, Firestein GS, van den Berg WB, Miossec P: Role of interleukin 17 in arthritis chronicity through survival of synoviocytes via regulation of synoviolin expression. PLoS One 2010, 5:e13416

56. McBee ME, Zeng Y, Parry N, Nagler CR, Tannenbaum SR, Schauer DB: Multivariate modeling identifies neutrophil- and Th17-related factors as differential serum biomarkers of chronic murine colitis. PLoS One 2010, 5:e13277

57. Gordon S: Alternative activation of macrophages. Nat Rev Immunol 2003, 3:23-35

58. Hesse M, Modolell M, La Flamme AC, Schito M, Fuentes JM, Cheever AW, Pearce EJ, Wynn TA: Differential regulation of nitric oxide synthase- 2 and arginase- 1 by type 1/type 2 cytokines in vivo: granulomatous pathology is shaped by the pattern of L-arginine metabolism. J Immunol 2001, 167:6533-6544

59. Honey K, Rudensky AY: Lysosomal cysteine proteases regulate antigen presentation. Nat Rev Immunol 2003, 3:472-482

60. Boring L, Gosling J, Chensue SW, Kunkel SL, Farese RV Jr, Broxmeyer $\mathrm{HE}$, Charo IF: Impaired monocyte migration and reduced type 1 (Th1) cytokine responses in C-C chemokine receptor 2 knockout mice. J Clin Invest 1997, 100:2552-2561

61. Lu B, Rutledge BJ, Gu L, Fiorillo J, Lukacs NW, Kunkel SL, North R, Gerard C, Rollins BJ: Abnormalities in monocyte recruitment and cytokine expression in monocyte chemoattractant protein 1-deficient mice. J Exp Med 1998, 187:601-608

62. Xiong M, Elson G, Legarda D, Leibovich SJ: Production of vascular endothelial growth factor by murine macrophages: regulation by hyp- 
oxia, lactate, and the inducible nitric oxide synthase pathway. Am $J$ Pathol 1998, 153:587-598

63. Wu WK, Llewellyn OP, Bates DO, Nicholson LB, Dick AD: IL-10 regulation of macrophage VEGF production is dependent on macrophage polarisation and hypoxia. Immunobiology 2010, 215:796-803

64. Friedlander M: Fibrosis and diseases of the eye. J Clin Invest 2007 117:576-586

65. Forrester JV, Xu H, Lambe T, Cornall R: Immune privilege or privileged immunity? Mucosal Immunol 2008, 1:372-381
66. Ferrari G, Cook BD, Terushkin V, Pintucci G, Mignatti P: Transforming growth factor-beta 1 (TGF-beta1) induces angiogenesis through vascular endothelial growth factor (VEGF)-mediated apoptosis. J Cell Physiol 2009, 219:449-458

67. Lux A, Salway F, Dressman HK, Kroner-Lux G, Hafner M, Day PJ, Marchuk DA, Garland J: ALK1 signalling analysis identifies angiogenesis related genes and reveals disparity between TGF-beta and constitutively active receptor induced gene expression. BMC Cardiovasc Disord 2006, 6:13 